\title{
ESTUDIO MORFOLÓGICO Y ESTRUCTURAL DE NANOPARTÍCULAS DE CUO EMPLEANDO TÉCNICAS DE DISPERSIÓN DE LUZ Y RAYOS X
}

\author{
G. Ríos ${ }^{\mathrm{a}}$, C. Giannini ${ }^{\mathrm{b}}$, D. Siliqi ${ }^{\mathrm{b}}$, D. Altamura ${ }^{\mathrm{b}}$, T. Sibillano ${ }^{\mathrm{b}}$, J.L. Solís ${ }^{\mathrm{a}}$, M.M. Gómez ${ }^{* a}$
}

\begin{abstract}
RESUMEN
Nanopartículas esféricas de óxido de cobre $(\mathrm{CuO})$ fueron sintetizadas por vía húmeda partiendo de acetato de cobre e hidróxido de sodio. Las nanopartículas de $\mathrm{CuO}$ fueron caracterizadas por dispersión dinámica de luz (DLS de los términos en inglés Dynamic Light Scattering) y por dispersión de rayos X en ángulos pequeños (SAXS de los términos en inglés Small Angle X-Ray Scattering). Además, para completar el estudio estructural y morfológico de las nanopartículas se hicieron análisis de difracción de rayos X y microscopía electrónica de barrido por emisión de campo. Los resultados mostraron que las nanopartículas de $\mathrm{CuO}$ presentaron alta dispersión. Sin embargo, estrecha distribución de tamaños en las poblaciones de tamaños menores de $100 \mathrm{~nm}$. Además se observó muy buena concordancia entre las medidas de dispersión de luz y rayos $\mathrm{X}$, así como con difracción de rayos $\mathrm{X}$ y microscopía electrónica.
\end{abstract}

Palabras clave: $\mathrm{CuO}$, nanopartículas, dispersión dinámica de luz (DLS), dispersión de rayos $\mathrm{X}$ en ángulos pequeños (SAXS).

\begin{abstract}
Spherical cooper oxide $(\mathrm{CuO})$ nanoparticles were synthetized by wet route using cooper acetate and sodium hydroxide. The $\mathrm{CuO}$ nanoparticles were characterized by dynamic light scattering (DLS) and small angle X-ray scattering (SAXS). Furthermore to complete structural and morphological studies of the nanoparticles were performed X-ray diffraction and electron microscopy analysis. The results displayed that the $\mathrm{CuO}$ nanoparticles showed high dispersion; however, has a narrow size distribution for particles with size less than $100 \mathrm{~nm}$. Besides, the correlation between dispersion analysis, light and X-ray, and X-ray diffraction and electron microscopy was very good.

Key words: $\mathrm{CuO}$, nanoparticles, dynamic light scattering (DLS), small angle X-ray scattering, X-ray diffraction (SAXS).

\section{INTRODUCCIÓN}

Actualmente, las nanopartículas de $\mathrm{CuO}$ poseen un gran interés debido a sus novedosas propiedades y su amplio potencial para aplicaciones catalíticas, fotocatalíticas, energéticas (como su uso en celdas solares y electrodos para baterías), electrónicas (para uso en sensores de gases) y ambientales (materiales antimicrobiales). ${ }^{1-5}$ Para toda esta gama de aplicaciones es de gran importancia obtener polvos constituidos de nanopartículas de alta calidad y ultra finos, con características específicas requeridas en términos de tamaño, morfología, microestructura, pureza, etc; dado que, el conjunto de todas estas características determinan el rendimiento de los productos finales.
\end{abstract}

Algunos métodos para la preparación de nanopartículas de $\mathrm{CuO}$ han sido reportados, tales

a Facultad de Ciencias, Universidad Nacional de ingeniería, Av. Túpac Amaru 210, Lima25, Perú mgomez@uni.edu.pe

b Institute of Crystallography, Italian National Research Council, Via Amendola 122/O, Bari, Italia 
como el método de precipitación rápida,,${ }^{6,7}$ molienda mecánica de polvos comerciales, ${ }^{8}$ el método sonoquímico, ${ }^{9}$ método de reacción de estado sólido de un solo paso a temperatura ambiental, ${ }^{10}$ método de rociado pirolítico asistido con ultrasonido, ${ }^{11}$ etc. Entre las diferentes técnicas de síntesis desarrolladas durante los últimos años, el método de precipitación rápida de nanopartículas de $\mathrm{CuO}$ es un método con gran potencial porque es simple, económico, seguro y eficiente.

\section{PARTE EXPERIMENTAL}

\section{Síntesis de las nanopartículas de $\mathrm{CuO}$}

La síntesis de las nanopartículas de $\mathrm{CuO}$ se realizó empleando $250 \mathrm{ml}$ de una solución acuosa 0,020 M de acetato de cobre monohidratado, $\mathrm{Cu}(\mathrm{CH} 3 \mathrm{COO}) \_2 . \mathrm{H} \_2 \mathrm{O}$, a la cual se le agregó, gota a gota ácido acético glacial, $\mathrm{CH}_{3} \mathrm{COOH}$, hasta alcanzar un $\mathrm{pH} \sim 4$. Posteriormente la solución fue simultáneamente agitada durante 3 min a $400 \mathrm{rpm}$ y calentada hasta una temperatura cercana a los $100^{\circ} \mathrm{C}$. Luego se agregó $1,5 \mathrm{ml}$ de una solución de hidróxido de sodio $11 \mathrm{M}$. La reacción química que representa el proceso de síntesis del $\mathrm{CuO}$ es:

$$
\mathrm{Cu}\left(\mathrm{CH}_{3} \mathrm{COO}\right)_{2(a c)}+2 \mathrm{NaOH}_{(a c)} \stackrel{\Delta}{\rightarrow} \mathrm{CuO}_{(s)}+2 \mathrm{NaCH}_{3} \mathrm{COO}_{(a c)}+\mathrm{H}_{2} \mathrm{O}_{(l)}
$$

Para el lavado del precipitado de $\mathrm{CuO}$ marrón oscuro, se eliminó el líquido sobrenadante y se agregó agua destilada; se agitó y se procedió a centrifugar para separar nuevamente el precipitado.

Los análisis de la morfología de las nanopartículas por medio de las técnicas de dispersión se desarrollaron empleando dos suspensiones acuosa de nanopartículas (muestras 1 y 2) a una concentración $1 \mathrm{mg} / \mathrm{ml}$ a un $\mathrm{pH} \sim 6$.

Para los análisis estructurales y morfológicos por medio de difracción de rayos X y microscopía electrónica, respectivamente, las nanopartículas se secaron a $80^{\circ} \mathrm{C}$ durante 2 horas.

\section{Análisis de las nanopartículas de $\mathrm{CuO}$}

\section{RESULTADOS Y DISCUSIÓN}

La caracterización estructural y morfológica de un material nanoparticulado es un factor determinante para entender sus propiedades, no solo físicas sino también químicas, y en base a ello explorar diversas aplicaciones tecnológicas que permitan obtener un mayor rendimiento de sistemas energéticos, mecánicos, ambientales, etc.

En esta sección presentamos el estudio morfológico de las nanopartículas de $\mathrm{CuO}$ mediante técnicas de dispersión de luz y de rayos X. Finalmente, estos análisis han sido complementados con estudios de difracción de rayos $\mathrm{X}$ y microscopía electrónica.

\section{Dispersión de luz}

Para desarrollar esta caracterización se empleó un equipo analizador de partículas Brookhaven Instruments 90Plus Nanoparticle Size Analizer. 
Previo a cada medida, las suspensiones de las nanopartículas de $\mathrm{CuO}$ fueron sonicadas durante $2 \mathrm{~min}$. Cada medida consistió en tres corridas de $20 \mathrm{~s}$ cada una. Se empleó un láser de longitud de onda $(\lambda)$ de $658 \mathrm{~nm}$ a un ángulo de detección (dispersión) de $90^{\circ}$. Se consideró a las partículas de $\mathrm{CuO}$ (índice de refracción $n_{\mathrm{CuO}}=2,741+0,389 \mathrm{i}$ ) como esféricas difundiéndose con movimiento Browniano en agua (índice de refracción $n_{\mathrm{H} 2 \mathrm{O}}=1,33 \mathrm{y}$ viscosidad $0,890 \mathrm{cP})$ a una $\mathrm{T}=25^{\circ} \mathrm{C}(=298 \mathrm{~K})$.

La figura 1 muestra los histogramas para las dos muestras de $\mathrm{CuO}$ sintetizadas. La frecuencia relativa está denotada como Intensidad, la cual está definida como intensidad dispersada relativa de las poblaciones de partículas dispersadas por unidad de tamaño de clase.

$$
\text { Intensidad }=\left(\frac{100}{I_{0}}\right) \frac{d I}{d D}
$$

Donde $I_{0}$ es la intensidad dispersada de partículas dentro de la población total de partículas dispersadas, $d I$ es la intensidad de la población de partículas dispersadas de tamaño $d D$
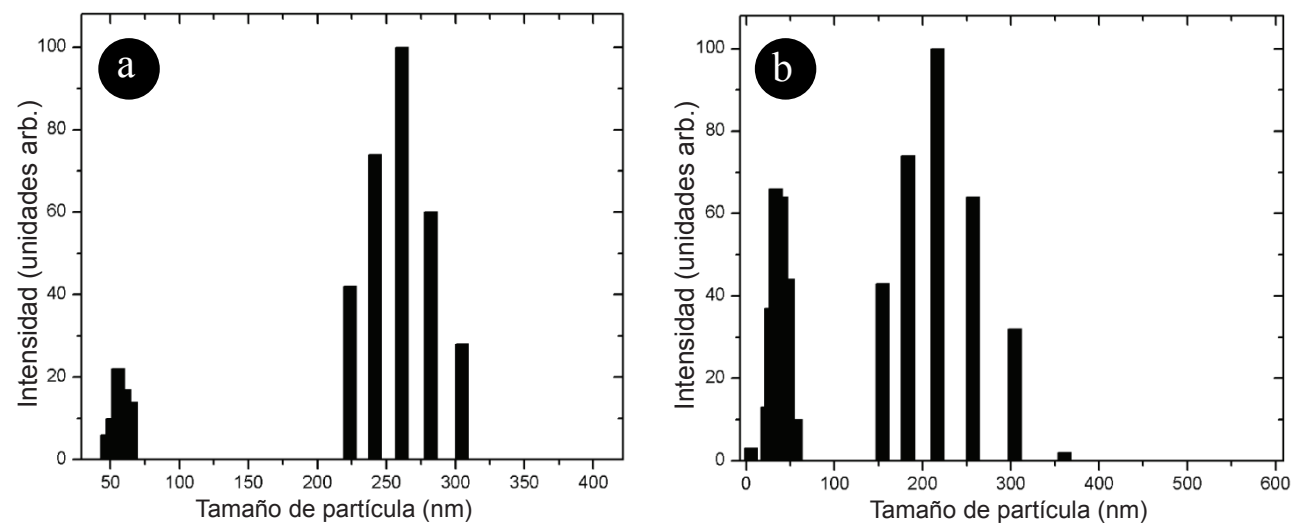

Figura 1. Distribución de tamaños de las nanopartículas del $\mathrm{CuO}$ respecto a la intensidad dispersada relativa para (a) muestra 1 y (b) muestra 2.

De las figuras 1a y $1 \mathrm{~b}$ se puede observar que ambas muestras presentan dos poblaciones de nanopartículas. Para el caso de la muestra 1 (figura 1a) una población está alrededor de 55 $\mathrm{nm}$ y otra alrededor de $261 \mathrm{~nm}$. Mientras que para el caso de la muestra 2 (figura 1b) las poblaciones presentaron los tamaños de $37 \mathrm{~nm}$ y $217 \mathrm{~nm}$.

\section{Dispersión de rayos $X$ en ángulos pequeños}

El equipo utilizado para estas medidas fue un sistema SAXS/WAXS del XMILab, equipado con fuente de ánodo rotante Rigaku Fr-E+ $(2,5 \mathrm{~kW}$ de potencia) de emisión $\mathrm{CuK}$ : $\lambda=$ $0,15405 \mathrm{~nm}$, acoplado a un arreglo óptico focalizante CMF 15-105, y cámara SMAX3000; la dispersión a ángulo pequeño se colectó en un detector multi-hilo (1024x1024 píxeles) 
puesto a 2,2 $\mathrm{m}$ de distancia de la muestra. El intervalo accesible en términos de vectores de dispersión fue entre 0,006 y $0,2 \AA^{-1}$, mientras que el rango angular accesible entre -1 y $+6^{\circ}$.

El tamaño del haz en la posición de las muestras fue de aproximadamente $200 \mu \mathrm{m}$ de diámetro; y se usó un bloqueador de haz de 5,5 $\mathrm{mm}$.

Previo a las medidas se empleó para la calibración del equipo un polvo estándar de $A g$ Behenate puesto en un capilar de $8 \mathrm{~mm}$ de diámetro y se midió su dispersión de rayos $\mathrm{X}$ con el objetivo de determinar la relación pixeles $\leftrightarrow$ vectores q.

Los análisis se realizaron para el solvente y las soluciones puestas en capilares de $8 \mathrm{~mm}$ en diferentes posiciones de cada capilar y para tiempo de colección de datos de $600 \mathrm{~s}$ y $1200 \mathrm{~s}$. La intensidad del haz incidente (la máxima intensidad transmitida sin muestra) fue medida dentro de la cámara por un detector adicional. La intensidad proveniente de la dispersión de rayos $\mathrm{X}$ fue medida por un detector $2 \mathrm{D}$ a 2,2 $\mathrm{m}$ lejos de la muestra.

La intensidad dispersada colectada fue la proveniente de la región central de cada capilar (para el solvente y cada suspensión solución). Similarmente, la intensidad transmitida fue medida en los mismos puntos investigados en el experimento SAXS, y el coeficiente de transmisión (T) fue calculado en cada punto como:

$$
T=\frac{I_{\text {Transmitido }}}{I_{0}}
$$

donde $I_{0}$ es la intensidad del haz primario; en los experimentos se ha considerado que Tsolución $\approx$ Tsolvente. La intensidad del Background (la intensidad total debido al solvente, aire dentro de la cámara, ventanas, etc., es decir, sin muestras) $I_{\text {Background }}$ fue entonces substraído de los datos de dispersión para obtener la intensidad de las partículas $I_{\text {Particulas }}$ solamente, tomando en cuenta los coeficientes de transmisión según:

$$
I_{\text {Partículas }}=I_{\text {medido }}-T \times I_{\text {Backgound }}
$$

La figura 2 muestra los perfiles de dispersión obtenidos directamente de los experimentos para (a) el solvente, (b) para la muestra 1 y (c) la muestra 2. El ángulo azimutal representado por $\alpha$ es el azimutal (no ángulo de dispersión) el cual está definido en el plano de detección como el ángulo en posición normal (trigonométrico) cuyo vértice coincide con el punto de impacto entre el haz de rayos X y la muestra. Para cada muestra se ha considerado varios puntos del capilar, pero por razones prácticas, en las siguientes figuras se está mostrando sólo un punto del capilar. 


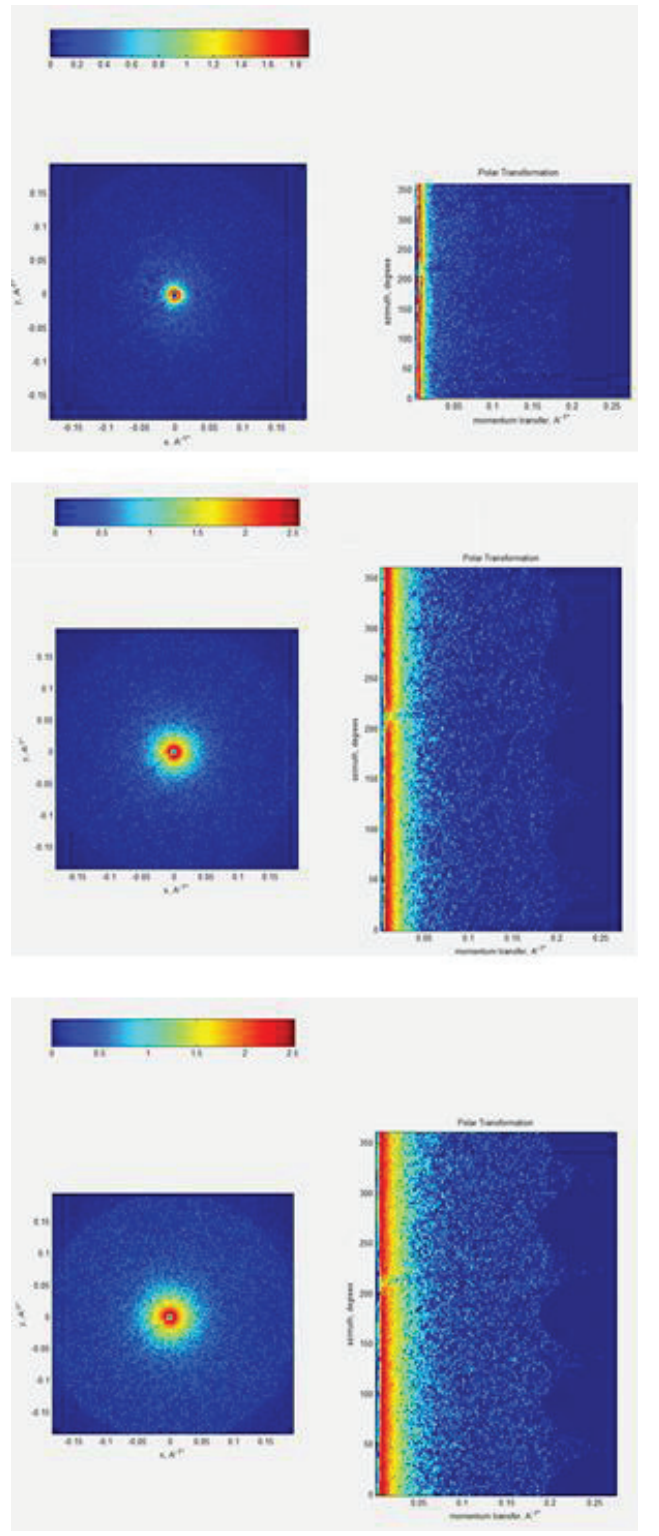

Figura 2. Perfil de dispersión de (a) la matriz o solvente (agua), (b) muestra 1 y (c) muestra 2, en un punto central del capilar (de coordenadas: $\mathrm{x}=22,5 \mathrm{~mm}, \mathrm{y}=0,016 \mathrm{~mm}$ ) con un tiempo de medición de 600 s y 1200 s. (Izquierda) En coordenadas cartesianas: $x=q_{x}, y=q_{y}$. (Derecha) En coordenadas polares: $\mathrm{x}=\mathrm{q}, \mathrm{y}=\alpha, \mathrm{q}=\sqrt{q_{x}{ }^{2}+q_{y}^{2}}, 0^{\circ}<\alpha<360^{\circ}, \alpha$ es el ángulo azimutal (ángulo central en posición normal del perfil de dispersión). La escala de grises corresponde a los valores de intensidad dispersada. Gris oscuro para intensidad: 0 , y blanco para intensidad: 2,5. La posición del bloqueador del haz debería ser mostrada en ausencia de dispersión (como una línea gris oscura) a un ángulo ' $\alpha$ ' ligeramente mayor que $200^{\circ}$ 
Comparando los resultados SAXS con los obtenidos por DLS se puede ver que, particularmente para la muestra 2 (partículas con diámetro alrededor de $30 \mathrm{~nm}$ en promedio), el diámetro promedio de partícula obtenido por SAXS está en buen acuerdo con el diámetro promedio de partícula en la intensidad vs. diámetro obtenido con DLS. Efectivamente, la configuración SAXS usada para el experimento no es muy sensible para partículas más grandes que $100 \mathrm{~nm}$ (a causa de los 2,2 $\mathrm{m}$ de distancia muestra-detector y los 5,5 $\mathrm{mm}$ de dimensión del bloqueador de haz). Así que la población de partículas con tamaños más grandes en cada muestra básicamente no es esperado que contribuya en la señal SAXS. Una comparación consistente entre los datos DLS y SAXS debería hacerse entonces considerando solamente la población de partículas de tamaño medio comprendido entre el rango común de 1-100nm. En el caso de la muestra 1 un diámetro promedio de partícula en el rango 33 - $36 \mathrm{~nm}$ fue obtenido de SAXS, el cual debería ser comparado con el diámetro promedio de $55 \mathrm{~nm}$ obtenido por DLS para la población de tamaños más pequeños. Esta discrepancia en tamaños puede ser explicada debido a que según DLS la concentración de nanopartículas más pequeñas en la muestra 1 es menor comparada con la muestra 2, sin embargo la medida de SAXS es independiente de la concentración y se realizaron medidas en varios puntos del capilar.

La tabla 1 muestra la comparación de los resultados obtenidos por ambas técnicas DLS y SAXS.

Tabla 1. Comparación de los resultados obtenidos por dispersión de luz (DLS) y dispersión de rayos X en ángulos pequeños (SAXS).

\begin{tabular}{ccc}
\hline Muestra de nanopartículas de & $\begin{array}{c}\text { Diámetro DLS } \\
(\mathrm{nm})\end{array}$ & $\begin{array}{c}\text { Diámetro SAXS } \\
\mathrm{CuO}\end{array}$ \\
1 & $\mathbf{5 7}$ & $\mathbf{n m})$ \\
2 & $\mathbf{3 7}$ & $\mathbf{3 4}$ \\
\hline
\end{tabular}

\section{Microscopía electrónica de barrido de emisión de campo}

La morfología de las nanopartículas fue investigada por microscopía electrónica de barrido por emisión de campo (FESEM de los términos en inglés Field Emission Scanning Electron Microscopy) usando un microscopio JEOL JSM-7100FT operado a un $1 \mathrm{kV}$ de voltaje. Las muestras también fueron analizadas a $30 \mathrm{kV}$ usando un detector STEM (Scanning Transmission Electron Microscope). Previo a cada análisis las nanopartículas de $\mathrm{CuO}$ fueron dispersadas en alcohol con sonicación durante unos minutos.

La figura 3 presenta las micrografías de nanopartículas de $\mathrm{CuO}$. Como se puede observar en la figura 3a, las nanopartículas están aglomeradas formando cúmulos de alrededor de $30 \mathrm{~nm}$ que, a su vez, forman extensiones largas de varios cientos de nanómetros. Estos cúmulos a su vez están constituidos por nanocristales que presentan un tamaño aproximado de $10 \mathrm{~nm}$ (Figura 3b). 

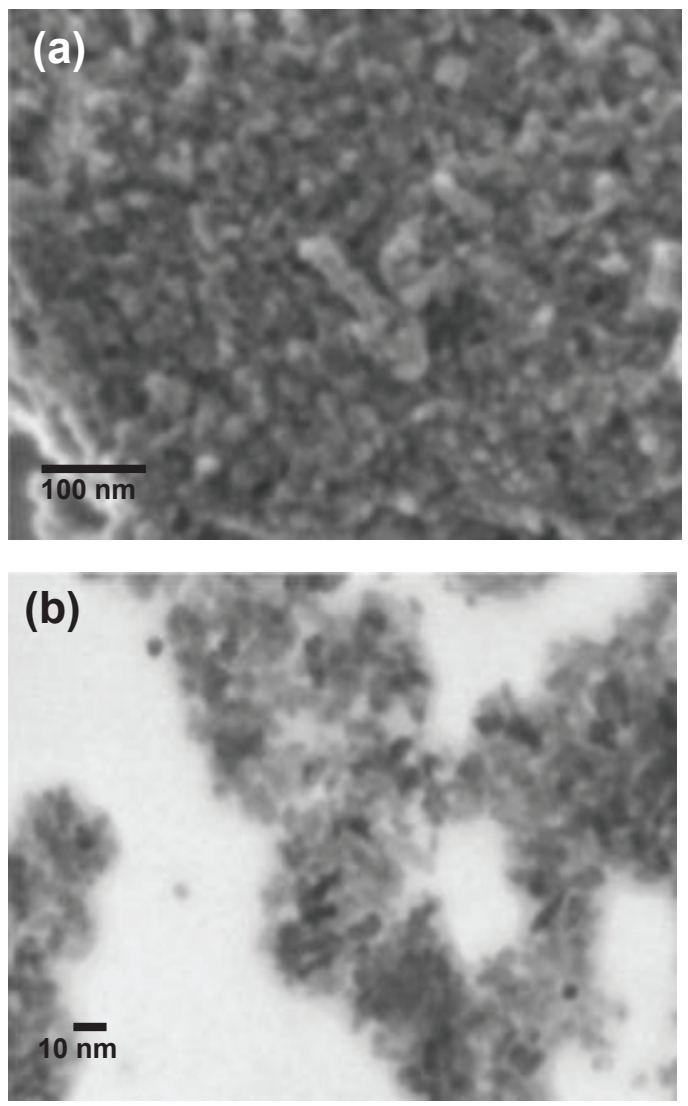

Figura 3. Micrografías electrónicas de nanopartículas de $\mathrm{CuO}$. (a) Imagen obtenida bajo la modalidad de SEM y (b) imagen obtenida en modo STEM.

\section{Difracción de rayos $\mathrm{X}$}

La estructura y tamaño de dominios cristalinos de las nanopartículas de $\mathrm{CuO}$ fueron determinadas por difracción de rayos X usando un difractómetro Rigaku Miniflex II Desktop operando con radiación $\mathrm{CuK}_{\alpha}(\lambda=0,15045 \mathrm{~nm})$ a $30 \mathrm{kV}$ y $20 \mathrm{~mA}$ con una velocidad de barrido de ángulo de $3 \mathrm{deg} \mathrm{min}^{-1}$. Para la determinación del tamaño de cristalino de las nanopartículas los datos de difracción fueron analizados con un proceso general de convolución (TopasAcademic) permitiendo, en principio, cualquier combinación de funciones apropiadas para ser empleadas para modelar el perfil del difractograma del $\mathrm{CuO}$ obtenido. ${ }^{12}$

La figura 4 muestra el difractograma obtenido experimentalmente y el análisis efectuado con el programa Topas. Todos los picos pudieron ser indexados perteneciendo a la fase tenorita de acuerdo a la base de datos JCPDS número 45-0937. Los tamaños de los dominios cristalinos fueron determinados a partir del ancho de las líneas (picos) de difracción de 
rayos $\mathrm{X}$ asumiendo una función Voigt. ${ }^{13} \mathrm{El}$ tamaño del dominio cristalino promedio para las nanopartículas de $\mathrm{CuO}$ fue $\sim 8 \mathrm{~nm}$.

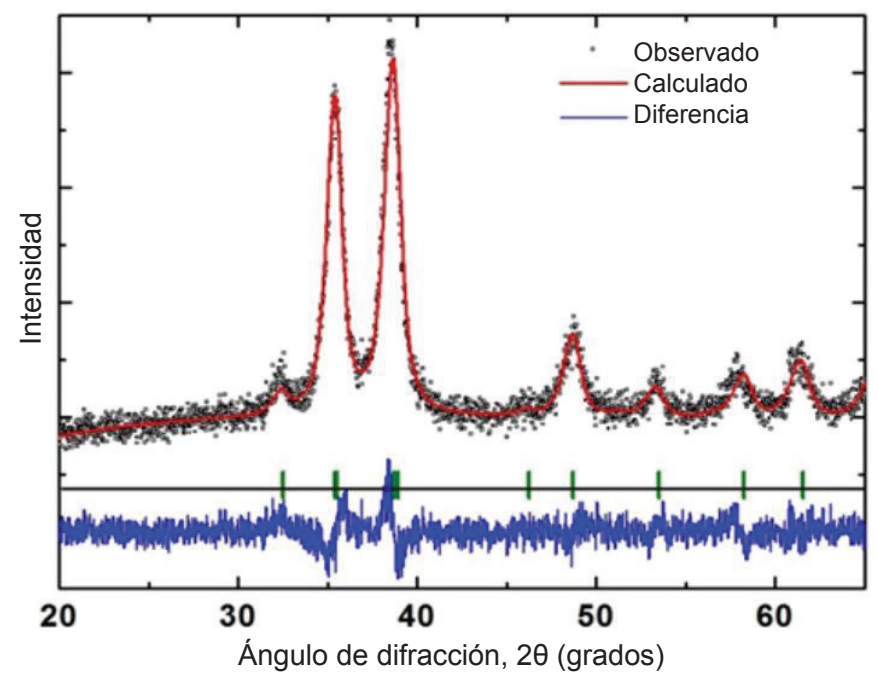

Figura 4. Patrón de difracción de rayos $\mathrm{X}$ de nanopartículas de $\mathrm{CuO}$.

\section{CONCLUSIONES}

Las muestras de $\mathrm{CuO}$ sintetizadas por precipitación rápida están formadas estructuralmente por nanopartículas constituidas por una red monoclínica de base centrada, con dominios cristalinos de $\sim 8 \mathrm{~nm}$ de tamaño en promedio. Estos cristalitos formaron principalmente conglomerados de partículas esféricas de $30 \mathrm{~nm}$ de diámetro promedio y también cúmulos largos de aproximadamente $102 \mathrm{~nm}$. Las técnicas de dispersión de luz (DLS) y rayos X en ángulos pequeños (SAXS) fueron empleadas para determinar su morfología y tamaño, y se encontró muy buena concordancia en los resultados obtenidos entre ambas.

\section{AGRADECIMIENTO}

Uno de nosotros (G.R.) agradece al Instituto General de Investigación de la Universidad Nacional de Ingeniería (IGI-UNI) por la beca otorgada.

El presente trabajo fue desarrollado bajo el auspicio de la Cooperación Científica Bilateral entre la CNR (Consiglio Nazionale delle Ricerche - Italia) y el CONCYTEC (Consejo Nacional de Ciencia y Tecnología - Perú).

\section{BIBLIOGRAFÍA}

1. Ben-Moshe T, Dror I, Berkowitz B. Oxidation of organic pollutants in aqueous solutions by nanosized copper oxide catalysts. Applied Catalysis B: Environmental. 2009;85(34):207-11. 
2. Musa AO, Akomolafe T, Carter MJ. Production of cuprous oxide, a solar cell material, by thermal oxidation and a study of its physical and electrical properties. Solar Energy Materials and Solar Cells. 1998;51(3-4):305-16.

3. Bandara J, Guasaquillo I, Bowen P, Soare L, Jardim WF, Kiwi J. Photocatalytic Storing of $\mathrm{O} 2$ as $\mathrm{H} 2 \mathrm{O} 2$ Mediated by High Surface Area CuO. Evidence for a ReductiveOxidative Interfacial Mechanism. Langmuir. 2005;21(18):8554-9.

4. Shishiyanu ST, Shishiyanu TS, Lupan OI. Novel NO2 gas sensor based on cuprous oxide thin films. Sensors and Actuators B: Chemical. 2006;113(1):468-76.

5. Podhajecky P, Scrosati B. Copper oxide cathodes for lithium organic electrolyte batteries. Journal of Power Sources. 1985;16(4):309-17.

6. Zhu J, Li D, Chen H, Yang X, Lu L, Wang X. Highly dispersed CuO nanoparticles prepared by a novel quick-precipitation method. Materials Letters. 2004;58(26):3324-7.

7. Lanje AS, Sharma SJ, Pode RB, Ningthoujam RS. Synthesis and optical characterization of copper oxide nanoparticles. Adv Appl Sci Res. 2010;1(2):36-40.

8. Bianchi AE, Stewart SJ, Punte G, Viña R, Plivelic TS, Torriani IL. Grain growth of $\mathrm{CuO}$ nanocrystal activated by high energy ball milling. Physica B: Condensed Matter. 2007;389(1):135-9.

9. Deng C, Hu H, Ge X, Han C, Zhao D, Shao G. One-pot sonochemical fabrication of hierarchical hollow $\mathrm{CuO}$ submicrospheres. Ultrasonics Sonochemistry. 2011;18(5):9327.

10. Xu JF, Ji W, Shen ZX, Tang SH, Ye XR, Jia DZ, et al. Preparation and Characterization of CuO Nanocrystals. Journal of Solid State Chemistry. 1999;147(2):516-9.

11. Oh SW, Bang HJ, Bae YC, Sun Y-K. Effect of calcination temperature on morphology, crystallinity and electrochemical properties of nano-crystalline metal oxides (Co3O4, $\mathrm{CuO}$, and $\mathrm{NiO}$ ) prepared via ultrasonic spray pyrolysis. Journal of Power Sources. 2007; 173(1):502-9.

12. Cheary RW, Coelho AA, Cline JP. Fundamental Parameters Line Profile Fitting in Laboratory Diffractometers. J Res Natl Inst Stand Technol. 2004;109:1-25.

13. Cheary RW, Coelho A. A Fundamental Parameters Approach to X-ray Line-Profile Fitting. J Appl Cryst. 1992;25:109-21. 